\title{
Large and tunable negative refractive index via electromagnetically induced chirality in a semiconductor quantum well nanostructure
}

\author{
Shun-Cai Zhao, ${ }^{1, *}$ Shuang-Ying Zhang, ${ }^{1}$ and You-Yang $\mathrm{Xu}{ }^{1}$ \\ ${ }^{1}$ Physics department, Kunming University of Science and Technology, Kunming, 650500, PR China
}

(Dated: December 20, 2018)

\begin{abstract}
Large and tunable negative refractive index (NRI) via electromagnetically induced chirality is demonstrated in a semiconductor quantum wells (SQWs) nanostructure by using the reported experimental parameters in Ref.[19]. It is found: the large and controllable NRI with alterable frequency regions is obtained when the coupling laser field and the relative phase are modulated, which will increase the flexibility and possibility of implementing NRI in the SQWs nanostructure. The scheme rooted in the experimental results may lead a new avenue to NRI material in solid-state nanostructure.
\end{abstract}

\section{INTRODUCTION}

In recent years there has been a huge interest in semiconductor quantum wells(SQWs), on account of the intersubband transitions (ISBTs) in SQWs are believed to have great potential applications in solid-state optoelectronics and quantum information science[1-4]. In many case the characteristics of ISBT dephasing mechanisms in SQWs behave essentially as "artificial atoms", which allowed us to regard the SQW as a single quantum object for many kinds of quantum optical phenomena, in which the nonlinear quantum optical phenomena have been extensively discussed, such as Kerr nonlinearity[5], ultrafast all optical switching[6], coherent population trapping[7], electromagnetically induced transparency [8-10], gain without inversion[11], enhancing index of refraction[12] and other novel phenomena [13-18]. But not only that, the devices based on ISBTs in SQWs also have many inherent advantages that the atomic systems don't have, such as the high nonlinear optical coefficients, the large transition energies and electric dipole moments for the small effective electron mass, the great flexibilities in symmetries design as well as in devices design by choosing the materials and structure dimensions.

\section{THEORETICAL MODEL}

In this work, we investigate the refractive index in a bulk SQW nanostructure via electromagnetically induced chirality. And the results demonstrate the large and tunable NRI can be realized by the coupling

\footnotetext{
*zhaosc@kmust.edu.cn
}

laser field and the relative phase. The SQW samples we simulate here are reported to be grown by the molecular beam epitaxy (MBE) method and each contains a $4.8 \mathrm{~nm} I n_{0.47} G a_{0.53} A s / 0.2 \mathrm{~nm} A l_{0.48} I n_{0.52} A s /$ $4.8 \mathrm{~nm} I n_{0.47} G a_{0.53} A s$ coupled quantum well, separated by a $36 \mathrm{~nm} A l_{0.48} I n_{0.52} A s$ modulation-doped barriers[19, 20]. And the transition energies of the ISBTs were measured as $\omega_{12}=124 \mathrm{meV}, \omega_{23}=185 \mathrm{meV}$, implying $\omega_{13}=309$ $\mathrm{meV}[19]$. The corresponding transition dipoles were calculated as $d_{12}=2.335 \mathrm{~nm}, d_{23}=2.341 \mathrm{~nm}$, and $d_{13}=0.120$ $\mathrm{nm}[21]$. So the synthesized 3-level cascade electronic system of ISBTs in such SQW forms a familiar ladder configuration, as shown in Fig.1.

FIG. 1. Fig.1 Schematic diagram of the SQW structure. The electric-dipole transition $|1\rangle-|3\rangle$ is driven by the coupling laser field, and the level pairs $|1\rangle-|2\rangle$ and $|3\rangle-|2\rangle$ are coupled to the electric and magnetic fields of the weak probe light, respectively.

The equivalent 3-level loop " atom" system simulated in our work is mainly based on the above reported SQW sample. In such a 3-level system, the parity of level $|1\rangle$ is set to be opposite to those of the levels $|2\rangle$ and $|3\rangle$, which have the same parity. The possible optical transition $|1\rangle-|3\rangle$ is mediated by a coupling laser field with central frequency $\omega_{c}$ and Rabi frequency $\Omega_{c}$. A weak probe laser field with central frequency $\omega_{p}$ and Rabi fre- 
quency $\Omega_{p e}$ is applied to the ISBT $|1\rangle-|2\rangle$. Because of the parity selection rules, the two levels $|1\rangle$ and $|2\rangle$ with electric dipole element $d_{12}=\langle 2|\hat{\vec{d}}| 1\rangle \neq 0$ are coupled by the electric component of the weak probe field, where $\hat{\vec{d}}$ is the electric dipole operator. The two levels $|2\rangle$ and $|3\rangle$ with the magnetic dipole element $\mu_{23}=\langle 2|\hat{\vec{\mu}}| 3\rangle \neq 0$ are coupled by the magnetic component of the probe field with Larmor frequency $\Omega_{p b}=\vec{B}_{p} \mu_{23} / \hbar$, where $\hat{\vec{\mu}}$ is the magnetic-dipole operator. The coherent cross-coupling between electric and magnetic dipole transitions driven by the electric and magnetic components of the probe field may lead to chirality[25, 26]. Because the transition $|1\rangle-|3\rangle$ is mediated by a coupling laser field, the transitions $|1\rangle-|2\rangle$ and $|3\rangle-|2\rangle$ are coupled respectively by the electric and magnetic components of the probe field; it then forms a closed-loop system. It is well known that in a loop configuration the dynamics behaviour becomes quite sensitive to phases[22].

Under the rotating-wave approximations in the interaction representation, with the assumption of $\hbar=1$ and employing the quantum regression theorem and the established treatment for 3-level atoms[23], the resulting Hamiltonian of the three-level configuration can be written as

$$
\begin{aligned}
H_{i n t}= & \Delta_{p e}|2\rangle\left\langle 2\left|+\left(\Delta_{c}+\Delta_{p b}\right)\right| 3\right\rangle\langle 3|-\left(\Omega_{p e} \exp \left(-i \varphi_{p e}\right)\right. \\
& |2\rangle\left\langle 1\left|+\Omega_{p b} \exp \left(-i \varphi_{p b}\right)\right| 3\right\rangle\langle 2|+\Omega_{c} \\
& \left.\exp \left(-i \varphi_{c}\right)|3\rangle\langle 1|+H . c\right),
\end{aligned}
$$

where $\Delta_{p e}=\omega_{21}-\omega_{p}, \Delta_{p b}=\omega_{23}-\omega_{p}$ and $\Delta_{c}=\omega_{13}-\omega_{c}$ are the ISBTs detunings of the corresponding fields, and $\Delta_{c}$ depicts the two photon detuning process. According to the measured transition energies in the above SQW sample[19], we can get the relationship $\Delta_{c}=\Delta_{p e}+\Delta_{p b}$, which can avert the major obstacle mentioned in Ref.[24] in realizing the predicted effects at a realistic experimental setting. The $\varphi_{p b}, \varphi_{p e}$ and $\varphi_{c}$ are denoted the relevant phases of the three coherent fields. The symbol H.c. means the Hermitian conjugate. Then the equation of the time-evolution for the system can be described as $\frac{d \rho}{d t}=-\frac{i}{\hbar}[H, \rho]+\Lambda \rho$, where $\Lambda \rho$ represents the irreversible decay part in the system. Under the dipole approximation the density matrix equations described the system are written as follows:

$$
\begin{aligned}
\dot{\rho_{22}=} & i \Omega_{p b}\left(\rho_{32}-\rho_{23}\right)+i \Omega_{p e}\left(\rho_{12}-\rho_{21}\right)-\gamma_{21} \rho_{22} \\
& +\gamma_{32} \rho_{33}, \\
\dot{\rho_{33}}= & i \Omega_{p b}\left(\rho_{32}-\rho_{23}\right)+i \Omega_{c} \exp (-i \phi) \rho_{13}-i \Omega_{c} \\
& \exp (i \phi) \rho_{31}-\left(\gamma_{32}+\gamma_{31}\right) \rho_{33}, \\
\rho_{12}= & i \Omega_{p e}\left(\rho_{22}-\rho_{11}\right)+i \Omega_{c} \exp (i \phi) \rho_{32}-i \Omega_{p b} \\
& \left(\rho_{13}+\left(i \Delta_{p e}-\frac{\Gamma_{12}}{2}\right) \rho_{12},\right. \\
\dot{\rho_{13}}= & i \Omega_{c} \exp (i \phi)\left(\rho_{33}-\rho_{11}\right)+i \Omega_{p e}\left(\rho_{23}-i \Omega_{p b}\right. \\
& \rho_{12}+\left[i\left(\Delta_{p e}+\Delta_{p b}\right)-\frac{\Gamma_{13}}{2}\right] \rho_{13}, \\
\rho_{23}= & i \Omega_{p b}\left(\rho_{33}-\rho_{22}\right)+i \Omega_{p e} \rho_{13}-i \Omega_{c}\left(\rho_{21}\right. \\
& +\left(i \Delta_{p b}-\frac{\Gamma_{23}}{2}\right) \rho_{23},
\end{aligned}
$$

where $\phi=\varphi_{c}-\varphi_{p e}-\varphi_{p b}$ is the relative phase of the three corresponding optical fields. And the above density matrix elements comply with the conditions: $\rho_{11}+$ $\rho_{22}+\rho_{33}=1$ and $\rho_{i j}=\rho_{j i}^{*}$. The total decay rates $\Gamma_{i j}$ are added phenomenologically[19, 25] in above density matrix equations, which include the population decay rates and dephasing decay rates. Among them, the population decay rates from ISBTs, denoted by $\gamma_{i j}$, are primarily due to longitudinal optical(LO) phonon emission events at low temperature. And the dephasing decay rates originate from electron-electron, interface roughness and phonon scattering processes. Thus the total decay rates $\Gamma_{i j}$ can been written by $\Gamma_{12}=\gamma_{12}+\gamma_{12}^{d p h}$, $\Gamma_{23}=\gamma_{31}+\gamma_{32}+\gamma_{21}+\gamma_{23}^{d p h}, \Gamma_{13}=\gamma_{31}+\gamma_{32}+\gamma_{13}^{d p h}$. When the probe field is weak, i.e. $\Omega_{p e}, \Omega_{p b} \ll \Omega_{c}, \Gamma_{i j}$, almost all the atoms can be assumed to be in the ground state|1 $\rangle$, the steady-state values of the density matrix elements $\rho_{12}$ and $\rho_{23}$ can be written in a linear approximation

$$
\begin{gathered}
\rho_{12}=\frac{2 A_{2} d_{12}}{i A_{1} A_{3}} \mathbf{E}+\frac{4 e^{-i \phi} \Omega_{c}\left(\Gamma_{23}+2 i \Delta_{p b}\right) u_{23}}{A_{1} A_{3}} \mathbf{B}, \\
\rho_{23}=\frac{4 e^{i \phi} A_{4} \Omega_{c} d_{12}}{A_{2} A_{3}^{*}} \mathbf{E}+\frac{8 u_{23} e^{2 i \phi} \Omega_{c}^{2}}{i A_{1}^{*} A_{3}^{*}} \mathbf{B}
\end{gathered}
$$

where $A_{i}^{*}(i=1,2,3,4)$ is the conjugate complex of $A_{i}$, and with $A_{1}=\Gamma_{12}\left(\Gamma_{23}+2 i \Delta_{p b}\right)-2 i \Gamma_{23} \Delta_{p e}+4\left(\Delta_{p e} \Delta_{p b}+\Omega_{c}^{2}\right)$, $A_{2}=\Gamma_{13}\left(\Gamma_{23}+2 i \Delta_{p b}\right)+\Gamma_{12}\left(\Gamma_{23}+2 i \Delta_{p b}\right)-A_{1}-4 \Delta_{p b}^{2}$, $A_{3}=\Gamma_{13}+2 i\left(\Delta_{p e}+\Delta_{p b}\right), A_{4}=\Gamma_{12}+\Gamma_{12}-2 i \Delta_{p b}$. The ensemble electric polarization and magnetization of the bulk SQWs to the probe field are given by $\vec{P}=N \overrightarrow{d_{12}} \rho_{21}$ and $\vec{M}=N \overrightarrow{\mu_{23}} \rho_{32}$, respectively, where $\mathrm{N}$ is the density of SQWs. Then the coherent cross-coupling between electric and magnetic dipole transitions driven by the electric and magnetic components of the probe field may 
lead to chirality[26, 27]. Substituting equations (3) and (4) into the formula for the ensemble electric polarization $\left(\vec{P}=N \overrightarrow{d_{12}} \rho_{21}\right)$ and magnetization $\left(\vec{M}=N \overrightarrow{\mu_{23}} \rho_{32}\right)$, we have the relations $\vec{P}=\alpha_{E E} \vec{E}+\alpha_{E B} \vec{B}, \vec{M}=\alpha_{B E} \vec{E}+$ $\alpha_{B B} \vec{B}$. Considering both electric and magnetic local field effects[28], $\vec{E}$ and $\vec{B}$ in $\vec{P}$ and $\vec{M}$ must be replaced by the local fields: $\vec{E}_{L}=\vec{E}+\frac{\vec{P}}{3 \varepsilon_{0}}, \vec{B}_{L}=\mu_{0}\left(\vec{H}+\frac{\vec{M}}{3}\right)$. Then we obtain

$$
\begin{aligned}
\vec{P}= & \frac{3 \varepsilon_{0}\left(\mu_{0} \alpha_{B B} \alpha_{E E}-\mu_{0} \alpha_{B E} \alpha_{E B}-3 \alpha_{E E}\right)}{B_{1}} \vec{E} \\
& +\frac{-9 \mu_{0} \varepsilon_{0} \alpha_{E B}}{B_{1}} \vec{H}, \\
\vec{M}= & \frac{3\left(\mu_{0} \alpha_{B E} \alpha_{E B}-\mu_{0} \alpha_{B B} \alpha_{E E}+3 \varepsilon_{0} \mu_{0} \alpha_{B B}\right)}{B_{2}} \vec{H} \\
& +\frac{9 \varepsilon_{0} \alpha_{B E}}{B_{2}} \vec{E}
\end{aligned}
$$

where $B_{1}=\mu_{0} \alpha_{B E} \alpha_{E B}+3 \alpha_{E E}-\mu_{0} \alpha_{B B} \alpha_{E E}-9 \varepsilon_{0}+$ $3 \mu_{0} \varepsilon_{0} \alpha_{B B}, \quad B_{2}=\mu_{0} \alpha_{B B} \alpha_{E E}+9 \varepsilon_{0}-\mu_{0} \alpha_{B E} \alpha_{E B}-$ $3 \alpha_{E E}-3 \varepsilon_{0} \mu_{0} \alpha_{B B}$ with $\alpha_{E E}=-2\left[\Gamma_{13}\left(\Gamma_{23}+2 i \Delta_{p b}\right)+\right.$ $\left.2 i \Gamma_{23}\left(\Delta_{p b}+\Delta_{p e}\right)-4\left(\Delta_{p b}^{2}+\Delta_{p b} \Delta_{p e}+\Omega_{c}^{2}\right)\right] d_{12} /\left[2\left(\Delta_{p e}+\right.\right.$ $\left.\left.\Delta_{p b}\right)-i \Gamma_{13}\right]\left[\Gamma_{12}\left(\Gamma_{23}+2 i \Delta_{p b}\right)-2 i \Gamma_{23} \Delta_{p e}+4\left(\Delta_{p e} \Delta_{p b}+\right.\right.$ $\left.\left.\Omega_{c}^{2}\right)\right] \hbar, \alpha_{E B}=4 e^{-i \phi}\left(\Gamma_{23}+2 i \Delta_{p b}\right) \Omega_{c} \mu_{23} /\left[\Gamma_{13}+2 i\left(\Delta_{p e}+\right.\right.$ $\left.\left.\Delta_{p b}\right)\right]\left[\Gamma_{12}\left(\Gamma_{23}+2 i \Delta_{p b}\right)-2 i \Gamma_{23} \Delta_{p e}+4\left(\Delta_{p e} \Delta_{p b}+\Omega_{c}^{2}\right)\right] \hbar$, $\alpha_{B E}=4 e^{i \phi}\left(\Gamma_{12}+\Gamma_{13}-2 i \Delta_{p b}\right) \Omega_{c} d_{12} /\left[\Gamma_{13}-2 i\left(\Delta_{p e}+\right.\right.$ $\left.\left.\Delta_{p b}\right)\right]\left[\Gamma_{12}\left(\Gamma_{23}-2 i \Delta_{p b}\right)+2 i \Gamma_{23} \Delta_{p e}+4\left(\Delta_{p e} \Delta_{p b}+\right.\right.$ $\left.\left.\Omega_{c}^{2}\right)\right] \hbar, \alpha_{B B}=8 e^{2 i \phi} \Omega_{c}^{2} \mu_{23} /\left[\Gamma_{13}-2 i\left(\Delta_{p e}+\Delta_{p b}\right)\right]\left[\Gamma_{12}\left(i \Gamma_{23}+\right.\right.$ $\left.\left.2 \Delta_{p b}\right)-2 \Gamma_{23} \Delta_{p e}+4 i\left(\Delta_{p e} \Delta_{p b}+\Omega_{c}^{2}\right)\right] \hbar$. The key idea of electromagnetic induced chirality is to use the magnetoelectric cross-coupling in which the electric polarization $\vec{P}$ is coupled to the magnetic field $\vec{H}$ of an electromagnetic wave and the magnetization $\vec{M}$ is coupled to the electric field $\vec{E}$ [27]:

$$
\vec{P}=\varepsilon_{0} \chi_{e} \vec{E}+\frac{\xi_{E H}}{c} \vec{H}, \vec{M}=\frac{\xi_{H E}}{c \mu_{0}} \vec{E}+\chi_{m} \vec{H}
$$

Here $\chi_{e}$ and $\chi_{m}, \xi_{E H}$ and $\xi_{H E}$ are the electric and magnetic susceptibilities, and the complex chirality coefficients, respectively. They lead to additional contributions to the refractive index for one circular polarization $[27,29]$ :

$$
n=\sqrt{\varepsilon \mu-\frac{\left(\xi_{E H}+\xi_{H E}\right)^{2}}{4}}+\frac{i}{2}\left(\xi_{E H}-\xi_{H E}\right)
$$

By comparison with equations (5) and (6), we obtain the permittivity by $\varepsilon=1+\chi_{e}$ and the permeability by $\mu=1+\chi_{m}$, and the complex chirality coefficients $\xi_{E H}$ and $\xi_{H E}$. In the above, we obtained the expressions for the electric permittivity and magnetic permeability of the bulk SQWs. Substituting equations from (3) to (6) into (7), the expression for refractive index can also be presented. In the section that follows, we will discuss the refractive index of the SQWs with the experimental parameters from Ref.[19].

\section{RESULTS AND DISCUSSION}

In this work, some key parameters are selected from the Ref.[19], which has the advantage than the photonicresonant materials because of the parameters used in the numerical simulation coming from the reported experimental results. The key parameters of the above mentioned SQWs sample are the measured transition energies[19], according to which the detunings of electricdipole and magnetic-dipole transitions are defined by the relation $\Delta_{p b}=\Delta_{p e}+61 \mathrm{mev}$, so we depict the two transitions to be different by setting $\Delta_{p b} \neq \Delta_{p e}$ (i.e. two transition frequencies are not near the same frequency in this SQWs system)[24]. In order to ensure linear response, the probe laser field is kept more than 100 times weaker to the coupling laser field. As far as the mentioned bulk SQWs sample be concerned, we estimate its average density in the cubic volume element as $\mathrm{N} \approx 1.04 \times 10^{22} \mathrm{~m}^{-3}$ from the mentioned experimental parameters. The parameter for the electric transition dipole moment from $|2\rangle \leftrightarrow|1\rangle$ is chosen from the measured parameter: $d_{12}=2.335 \times 1.602 \times 10^{-19} \mathrm{Cm}$, and the magnetic transition dipole moment is chosen from the typical parameter $\mu_{23}=7.0 \times 10^{-23} \mathrm{Cm}^{2} \mathrm{~s}^{-1}[30]$. Thus the total decay rates $\Gamma_{i j}$ are set $\Gamma_{i j}=5 \mathrm{mev}$ for all three ISBTs transitions from the measured parameters[19]. Another parameter is the coupling laser intensity, which should be below the damage threshold of SQWs. For its Rabi frequency, in this paper we choose the ranges of $\Omega_{c} \leq 20$ $\mathrm{meV}$. For example, when taking the maximal Rabi frequency $\Omega_{c}=20 \mathrm{meV}$ to make a calculation, the electric field amplitude is obtained $E \approx 10^{5} \mathrm{~V} / \mathrm{cm}$ according to the relationship $\hbar \Omega=\mu E$. Using of the connection between the electric field amplitude $\mathrm{E}$ and the intensity of the radiation $\mathrm{I}: \mathrm{E}=27.4682 \times \sqrt{I}$, we can get the intensity of the radiation $\mathrm{I}=13 \mathrm{MW} / \mathrm{cm}^{2}$, which shows the abovementioned laser intensities may be satisfied below the damage threshold of quantum object[31]. For the sake of simplification, we take the unit with $\varepsilon_{0}=\mu_{0}=1$.

Firstly, we discuss the refractive index dependence on the coupling laser field's Rabi frequences. We concentrate on the situation when the negative refraction is 
most prominent, i.e., under the condition of $\phi=0$. In Fig. 2, the plots of $\operatorname{Re}[\mathrm{n}]$ is shown as a function of $\Delta_{p e}$ with different $\Omega_{c}=9 \mathrm{mev}, 11 \mathrm{mev}, 13 \mathrm{mev}$ and $15 \mathrm{mev}$. And the dotted line, dashed dotted line, dashed line and solid line correspond to the four different values of $\Omega_{c}$, respectively. The dotted line has the most narrow interval of [-62.7mev, $58.3 \mathrm{mev}]$, in which the refraction index is negative and its maxima is -0.7 . The dashed dotted line shows its maxima of -1.32 in the interval [-64.5 mev, $-56.65 \mathrm{mev}$ ] for NRI. The maxima of NRI arrives to -2.3 in the interval [-64.0 mev, $-54.5 \mathrm{mev}$ ] when $\Omega_{c}$ was tuned to $13 \mathrm{mev}$. The interval expands to [-64.9 mev, -51.8 mev] in which the maxima of NRI is -3.7 when $\Omega_{c}=15$ mev. The increasing maxima of NRI in these expanding intervals is obtained by the gradually increasing $\Omega_{c}$, which can be explained via the quantum interference and coherence. The increasing coupling laser field drives the transition $|1\rangle-|3\rangle$ in the SQWs, which enhances interference between the electric dipole element and magnetic dipole element from the probe laser in ISBTs, then modifies the refractive index properties of the SQWs sample. The adjustable bandwidth for NRI in different frequency regions can also be drawn from equations (3) and (4). In equations (3), the coherent term $\rho_{12}$ is composed of two items: the former item is independent $\Omega_{c}$, while the latter cross-coupling item is proportional to $\Omega_{c}$ then be sensitive to $\Omega_{c}$. In the coherent term $\rho_{23}$, the two parts are proportional to $\Omega_{c}$ and $\Omega_{c}^{2}$, respectively. Then the induced chirality depends strongly on $\Omega_{c}$, which causes the refractive index being negative and the variable bandwidths.

FIG. 2. Fig. 2 The real parts of the refractive index $n$ as a function of the probe detuning $\Delta_{p e}$ with different Rabi frequencies $\Omega_{c}$ of coupling laser field: $9 \mathrm{mev}($ dotted line), 11 mev(dashed dotted line), $13 \mathrm{mev}$ (dashed line), $15 \mathrm{mev}$ (solid line). The parameters are: $\phi=0, \quad \Gamma_{12}=\Gamma_{13}=\Gamma_{23}=5 \mathrm{emv}$, $\Omega_{p e}=0.02 \mathrm{emv}, \Delta_{p b}=\Delta_{p e}+61 \mathrm{mev}$.

After studying the refractive index dependence on the coupling laser field's Rabi frequencies, we next study how the relative phase $\phi$ bring changes in the refractive index of the SQWs sample. As mentioned before, the dynamics behaviour is quite sensitive to phases[22] in a loop configuration, as can be see from equations (3) and (4). Equation (3) shows that the former coefficient in the coherent term $\rho_{12}$ is independent of the relative phase $\phi$, while the latter coefficient is sensitive to the relative phase $\phi$ through $e^{-i \phi}$. Similarly, equation (4) has the phase-dependent chirality expression before coefficient $\vec{E}$ through $e^{i \phi}$ and its second chirality part is dependent of the relative phase $\phi$ with $e^{2 i \phi}$. Thus, due to the presence of the induced chirality depends strongly on the relative phase $\phi$, which causes the refractive index to be negative via the adjusting relative phase.

FIG. 3. Fig.3 The real parts of the refractive index $n$ as a function of the probe detuning $\Delta_{p e}$ with different values of relative phase $\phi: 1.92 \pi$ (dotted line), $1.88 \pi$ (dashed dotted line), $1.86 \pi$ (dashed line) and $1.84 \pi$ (solid line), $\Omega_{c}=20 \mathrm{mev}$. The other parameters are the same as Fig.2.

In Fig. 3, the refraction index $\operatorname{Re}[\mathrm{n}]$ is plotted as a function of $\Delta_{p e}$ with the relative phase $\phi$ being $1.92 \pi$ (dotted line), $1.88 \pi$ (dashed dotted line), $1.86 \pi$ (dashed line) and $1.84 \pi$ (solid line) and $\Omega_{c}=20 \mathrm{mev}$. The sensitive adjustability via the relative phase $\phi$ is shown by the curves in Fig. 3. Interestingly, the Re[n] varies significantly with the different values of $\phi$ i.e., $\phi=1.92 \pi, 1.88 \pi, 1.86 \pi$ to $1.84 \pi$ when $\Omega_{c}=20 \mathrm{mev}$. The amplitudes of variation in the relative phase $\phi$ are $0.04 \pi$, $0.02 \pi$ and $0.02 \pi$ between the dotted line, dashed dotted line, dashed line and solid line, which causes the maximum of NRI changing from $-0.65,-1.78,-2.50$ to -3.45 , and the corresponding intervals enlarge from $[4.6 \mathrm{mev}$, $55.68 \mathrm{mev}]$, [0.48 mev, $55.68 \mathrm{mev}]$, [-0.78 mev, $55.68 \mathrm{mev}]$ to $[-1.70 \mathrm{mev}, 55.68 \mathrm{mev}]$. Hence, the bandwidths for NRI can also be adjusted by the relative phase. Comparing Fig.2 with Fig.3, the relative phase is much more sensitive than the coupling laser field in controlling the bandwidths for NRI. 


\section{CONCLUSION}

In summary, the large and tunable NRI is theoretically demonstrated in a SQWs nanostructure by using the reported experimental parameters. In our scheme, the coherent cross-coupling between electric and magnetic transitions leads to chirality and the chirality coefficients are modulated by the coupling laser field and relative phase, so the SQWs nanostructure can become NRI material. When the coupling laser field and relative phase are properly modulated, the large and tunable NRI with alterable frequency regions can be obtained. The flexibly modulating parameters and using reported experimental param- eters increase the possibility of implementing NRI in the SQWs nanostructure, which may give us a newt way to NRI material in solid state nanotructure. And we hope the coming experiment will achieve this in the future.

\section{ACKNOWLEDGMENT}

The work is supported by the National Natural Science Foundation of China (Grant No. 61205205)and the Foundation for Personnel training projects of Yunnan Province, China (Grant No. KKSY201207068).
[1] R. Tsu, L. L. Chang, G. A. Sai-Halasz, and L. Esaki, Phys. Rev. Lett. 341509 (1975).

[2] B. F. Levine, J. Appl. Phys. 74 R1 (1993).

[3] J. S. Yang, H. Sodabanlu, M. Sugiyama, Y. Nakano, and Y. Shimogaki, Appl. Phys. Lett. 95162111 (2009).

[4] H. Y. Zhu, T. Y. Zhang, and W. Zhao, J. Appl. Phys. 105043518 (2009).

[5] H. Sun, S. Gong, Y. Niu, S. Jin, R. Li, Z. Xu, Phys. Rev. B 74155314 (2006).

[6] J. H. Wu, J. Y. Gao, J. H. Xu, L. Silvestri, M. Artoni, G. C. LaRocca, F. Bassani, Phys. Rev. Lett. 95057401 (2005).

[7] J. F. Dynes, M. D. Frogley, J. Rodger, C. C. Phillips, Phys. Rev. B 72085323 (2005).

[8] L. Silvestri, F. Bassani, G. Czajkowski, B. Davoudi, Eur.Phys.J.B 2789 (2002).

[9] M. Phillips, H. Wang, Opt. Lett. 28831 (2003).

[10] A. Joshi, Phys. Rev. B 79115315 (2009).

[11] M. D. Frogley, J. F. Dynes, M. Beck, J. Faist, C. C. Phillips, Nat.Mater. 59175 (2006).

[12] S. M. Sadeghi, H. M. van Driel, J. M. Fraser, Phys. Rev. B 6215386 (2000).

[13] A. Joshi, M. Xiao, Appl.Phys.B 7965 (2004).

[14] J. F. Dynes, E. Paspalakis, Phys. Rev. B 73233305 (2006).

[15] J. H. Li, Phys. Rev. B 75155329 (2007).

[16] W. X. Yang, J. M. Hou, R. K. Lee, Phys. Rev. A 77 033838 (2008).

[17] M. D. Frogley, J. F. Dynes, M. Beck, J. Faist, C. C. Phillips, Nat.Mater. 59175 (2006).
[18] S. G. Kosionis, A. F. Terzis, E. Paspalakis, Appl. Phys. B 10433 (2011).

[19] J. F. Dynes, M. D. Frogley, M. Beck, J. Faist, C. C. Phillips, Phys. Rev. Lett. 94157403 (2005).

[20] Z. J. Simmons, N. A. Proite, J. Miles, D. E. Sikes, D. D. Yavuz, Phys. Rev. A 85053810 (2012).

[21] K. H. Yoo, L. R. Ram Mohan, and D. F. Nelson, Phys. Rev. B 3912808 (1989).

[22] D. V. Kosachiov, B. G. Matisov, and Y. V. Rodhestvensky, J. Phys. B: At Mol. Opt. Phys. 252473 (1992).

[23] L. M. Narducci, M. O. Scully, G.-L. Oppo, P. Ru and J. R. Tredicce, Phys. Rev. A 421630 (1990).

[24] M. Ö. Oktel, Ö. E. Mütecaplğu, Phys. Rev. A 70053806 (2004).

[25] G. B. Serapiglia, E. Paspalakis, C. Sirtori, K. L. Vodopyanov, C. C. Phillips, Phys. Rev. Lett. 841019 (2000).

[26] J. B. Pendry, Science 3061353 (2004).

[27] J. Käastel, M. Fleischhauer, R. L. Walsworth, Phys. Rev. A 79063818 (2009).

[28] X. M. Su, H. X. Kang, J. Kou, X. Z. Guo, and J. Y. Gao, Phys. Rev. A 8003805 (2009).

[29] F. L. Li, A. P. Fang, M. Wang, J. Phys. B: At Mol. Opt. Phys. 42199505 (2009).

[30] J. Q. Shen, J. Mod. Opt. 532195 (2006).

[31] C. J. Chang-Hasnain, P. C. Ku, J. Kim, S. L. Chuang, Proc. IEEE 911884 (2003). 


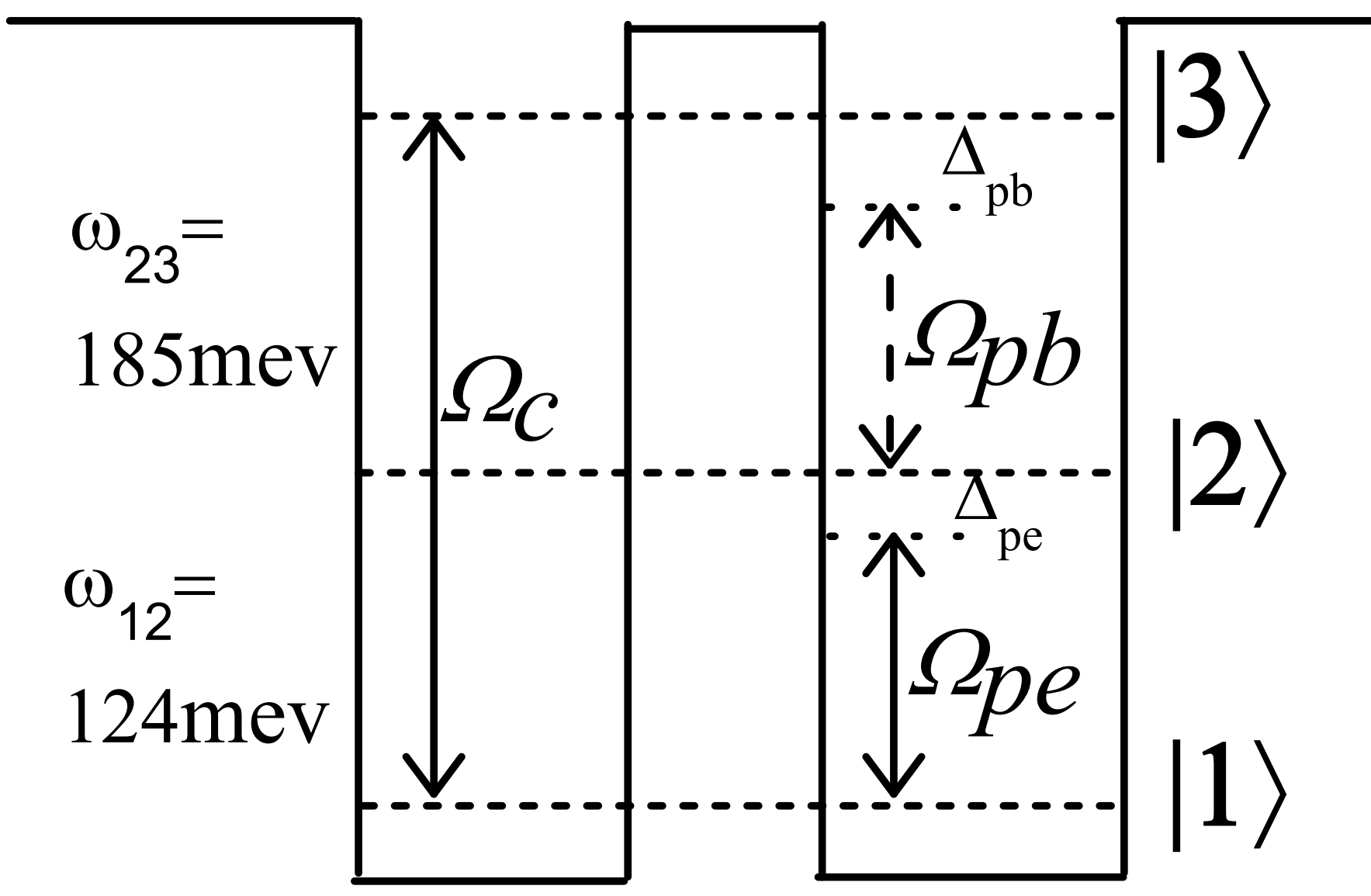

